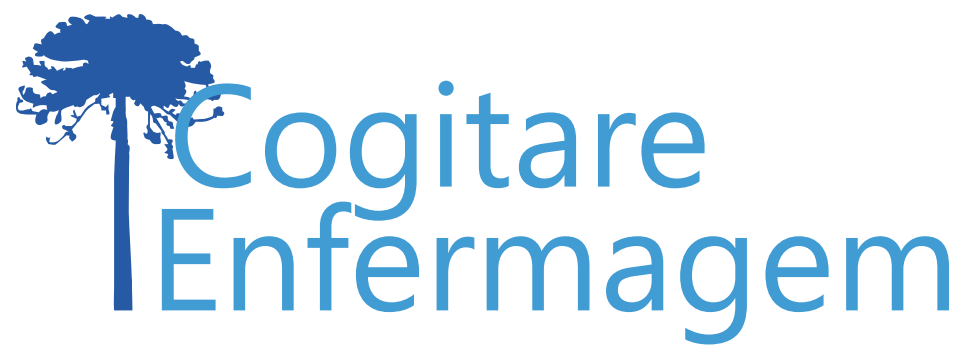

REVISÃO

\title{
CUIDADOS DE ENFERMAGEM NA TERMORREGULAÇÃO DE RECÉM-NASCIDOS PREMATUROS: REVISÃO INTEGRATIVA
}

Leilson da Silva Lima1, Erica Amoras Ferraz Reis², Eloisa Melo da Silva ${ }^{3}$, José Pedro Gomes Moura ${ }^{4}$

\section{RESUMO}

bjetivo: evidenciar e discutir os cuidados de enfermagem no que concerne à termorregulação de recém-nascidos prematuros.

Método: revisão integrativa com busca por artigos primários completos, publicados de 2014 a 2019, em português, inglês ou espanhol na Biblioteca Virtual de Saúde, PubMed e Cochrane Library. Utilizou-se os descritores: Termorregulação; Recém-Nascido; Enfermagem; "Enfermeiras e Enfermeiros"; "Body Temperature Regulation"; e "Infant, Newborn".

Resultados: foram incluídos nove artigos para síntese, com estudos realizados em diferentes países. Emergiram categorias sobre cuidados na termorregulação de recém-nascidos prematuros e sobre protocolos para a termorregulação neonatal na prática clínica.

Considerações finais: os cuidados de enfermagem na termorregulação incluem o uso de cama aquecida, aquecedor radiante, métodos de isolamento corporal e ajuste da temperatura ambiente. Este estudo contribui fornecendo base para pesquisas sobre a situação clínica de recém-nascidos e possíveis associações entre a assistência de enfermagem que poderiam comprometer a termorregulação neonatal.

DESCRITORES: Termorregulação; Recém-Nascido; Enfermagem; Assistência Perinatal; Hipotermia.

COMO REFERENCIAR ESTE ARTIGO:

Lima L da S, Reis EAF, Silva EM da, Moura JPG. Cuidados de enfermagem na termorregulação de recémnascidos prematuros: revisão integrativa. Cogitare enferm. [Internet]. 2020 [acesso em "colocar data de acesso, dia, mês abreviado e ano"]; 25. Disponível em: http://dx.doi.org/10.5380/ce.v25i0.70889.

${ }^{1}$ Enfermeiro. Mestrando em Ciências da Saúde. Universidade Federal do Amapá. Macapá, AP, Brasil. $\bigcirc$ ${ }^{2}$ Enfermeira. Especialista em Enfermagem Obstétrica e em Gestão e Docência do Ensino Superior. Hospital da Mulher Mãe Luzia. Macapá, AP, Brasil. $\cdot$

${ }^{3}$ Discente de enfermagem. Universidade Federal do Amapá. Macapá, AP, Brasil. 0

${ }^{4}$ Enfermeiro. Mestrando em Ciências da Saúde. Hospital da Mulher Mãe Luzia. Macapá, AP, Brasil. 


\title{
NURSING CARE IN THE THERMO-REGULATION OF PRETERM NEWBORNS: AN INTEGRATIVE REVIEW
}

\begin{abstract}
Objective: to evidence and discuss nursing care regarding thermo-regulation for pre-term newborns.

Method: An integrative review with search by complete primary articles, published from 2014 to 2019 in Portuguese, English or Spanish in the Virtual Health Library, PubMed, and the Cochrane Library. The following descriptors were used: Termorregulação; Recém-Nascido; Enfermagem; "Enfermeiras e Enfermeiros"; "Body Temperature Regulation"; and "Infant, Newborn".

Results: nine articles were included for synthesis, with studies conducted in different countries. Categories emerged about care in the thermo-regulation of pre-term newborns and on protocols for neonatal thermo-regulation in the clinical practice.

Final considerations: nursing care in thermo-regulation includes the use of a heated bed, radiant heater, body insulation methods, and adjustment of room temperature. This study contributes by providing a basis for research on the clinical situation of newborns and possible associations in nursing care that could compromise neonatal thermo-regulation.
\end{abstract}

DESCRIPTORS: Thermo-regulation; Newborn; Nursing; Perinatal care; Hypothermia.

\section{CUIDADOS DE ENFERMERÍA EN LA TERMORREGULACIÓN DE RECIEN NACIDOS PREMATUROS: REVISION INTEGRADORA}

\begin{abstract}
RESUMEN:
Objetivo: evidenciar y debatir los cuidados de enfermería en lo referente a la termorregulación de recién nacidos prematuros.

Método: revisión integradora con búsqueda por artículos primarios completos, publicados entre 2014 y 2019, en portugués, inglés o español en la Biblioteca Virtual de Salud, PubMed y la Cochrane Library. Se utilizaron los siguientes descriptores: Termorregulação; RecémNascido; Enfermagem; "Enfermeiras e Enfermeiros"; "Body Temperature Regulation"; e "Infant, Newborn".

Resultados: se incluyeron nueve artículos para la síntesis, con estudios realizados en diferentes países. Surgieron categorías sobre cuidados en la termorregulación de recién nacidos prematuros y sobre protocolos para la termorregulación neonatal en la práctica clínica.

Consideraciones finales: los cuidados de enfermería en la termorregulación incluyen el uso de una cama con calefacción, calefactor radiante, métodos de aislamiento corporal y ajuste de la temperatura ambiente. Este estudio contribuye con el aporte de una base para realizar investigaciones sobre la situación clínica de los recién nacidos y sus posibles asociaciones en la asistencia de enfermería que podrían poner en riesgo la termorregulación neonatal.
\end{abstract}

DESCRIPTORES: Termorregulación; Recién Nacido; Enfermería; Asistencia Perinatal; Hipotermia. 
A termorregulação é definida como uma função fisiológica capaz de controlar e manter um ambiente corporal neutro. Valor menor que $36,5^{\circ} \mathrm{C}$ ou maior que $37,5^{\circ} \mathrm{C}$ é fator de risco para a morbimortalidade neonatal, uma vez que agrava ou favorece distúrbios metabólicos, desconforto respiratório, enterocolite necrosante e hemorragia intracraniana ${ }^{(1)}$. A capacidade de manter a temperatura corporal estável frente às variações do ambiente é limitada em recém-nascidos (RN), sendo assim, o controle térmico depende da idade gestacional e pós-natal, do peso de nascimento e das condições clínicas do $\mathrm{RN}^{(2)}$.

O estresse causado pela exposição ao frio causa problemas fisiológicos e metabólicos, como a vasoconstrição pulmonar e periférica, que resulta em diminuição na captação pulmonar de oxigênio aos tecidos e intensa glicólise anaeróbica, podendo levar à acidose metabólica. Assim, quando o consumo de oxigênio aumenta em resposta à hipotermia, a frequência respiratória do RN também aumenta( ${ }^{(3)}$.

As principais dificuldades na termorregulação neonatal são relacionadas a: superfície relativamente grande em comparação ao peso; queratinização inadequada da pele; capacidade metabólica limitada para a produção de calor; pequena camada subcutânea de gordura (gordura marrom); isolamento térmico inadequado ${ }^{(4)}$. Como agravante nessa situação, ao nascer prematuramente, o RN perde semanas de estimulação no útero materno e os distúrbios da termorregulação podem ser uma das principais condições de risco(5).

A termorregulação ineficaz é uma das principais complicações que acometem o RN, acarretando em perda de calor em consequência da evaporação e da temperatura do ambiente externo(6); $50,3 \%$ dos RN de extremo baixo peso apresentam episódios e complicações relacionadas com a hipotermia. A falta de proteção térmica leva rapidamente à hipotermia, desse modo, estratégias de proteção térmica simples são viáveis nos níveis comunitário e institucionais em ambientes de recursos limitados ${ }^{(7)}$.

Com a implementação da atenção humanizada ao RN, são preconizadas intervenções que se referem à individualidade e integralidade do cuidado, garantia de tecnologia para recuperação e segurança do RN, acolhimento à família, estabelecimento do vínculo e apego, dentre outras ${ }^{(8)}$. Nesse contexto, o enfermeiro tem um papel singular, pois lida com situações emocionais difíceis, com a fragilidade de um RN extremo, com a morte, sentimentos de ansiedade e insegurança por parte dos familiares, logo, é fundamental possuir habilidade técnica, conhecimentos específicos e atualizados ${ }^{(9)}$.

A Portaria 2.068 de 2016 garante a importância da enfermagem como recurso humano, considerando a presença mínima de pelo menos dois profissionais de nível superior na assistência para cada 20 binốmios mãe-RN, preferencialmente com especialização em neonatologia/obstetrícia ou dois anos de experiência profissional na área. Além disso, sugere-se que, para cada oito binômios mãe-RN, deve haver um técnico de enfermagem ${ }^{(10)}$.

A relevância desta pesquisa para a assistência de enfermagem fundamenta-se na necessidade de aprofundar o conhecimento sobre o efeito do cuidado na termorregulação de neonatos, levando em consideração as tecnologias, ações, conhecimento e atitudes que podem ser tomadas. Assistência essa que o enfermeiro desenvolve com a participação da equipe multidisciplinar, da puérpera e familiares envolvidos no cuidado. Diante disso, a presente investigação teve como objetivo evidenciar e discutir os cuidados de enfermagem no que concerne à termorregulação de recém-nascidos prematuros. 
Trata-se de uma revisão integrativa (RI) da literatura cuja finalidade foi reunir e sintetizar os resultados de pesquisas sobre um delimitado tema, de maneira ordenada e sistemática. A RI contribui para o processo de sistematização e análise dos resultados, visando a compreensão de determinada temática, a partir de outros estudos independentes ${ }^{(11)}$.

Para a elaboração da presente revisão, foram seguidas as seguintes etapas: (1) identificação do tema e elaboração da questão norteadora; (2) busca ou amostragem na literatura; (3) coleta de dados; (4) análise crítica dos estudos incluídos; (5) interpretação dos resultados; e (6) apresentação da revisão/síntese do conhecimento ${ }^{(12)}$.

Para a etapa de elaboração da pergunta de pesquisa foi adotado a estratégia $P I C O$ (13), onde " $P$ " refere-se à População ou Problema, "I" à intervenção ou interesse, "C" Intervenção de comparação ou sem intervenção e "O" desfecho (outcomes). Dessa forma, no presente estudo definiu-se P: Recém-nascidos prematuros; I: Cuidados de Enfermagem; C: Não intervenção; O: Melhora na termorregulação. Conforme essa estratégia, a pergunta elaborada foi: "quais são as evidências cientificas dos cuidados de enfermagem para a melhora na termorregulação de recém-nascidos prematuros?".

Na etapa de busca na literatura, encontra-se a seleção de estudos mediantes critérios claros, sendo assim, foi utilizado como estratégia a Biblioteca Virtual de Saúde (BVS), além de busca adicional na PubMed e Cochrane Library. Para obtenção da amostra na BVS, utilizou-se os descritores: Termorregulação AND "Recém-Nascido" AND ("Enfermeiras e Enfermeiros" OR Enfermagem), conforme os Descritores em Ciências da Saúde (DECS), já na PubMed e Cochrane Library utilizou-se: "Body Temperature Regulation" AND "Infant, Newborn" através dos Medical Subject Headings (MeSH).

Para filtragem da busca, foram selecionados estudos com textos completos, nos idiomas português, inglês e espanhol, publicados entre os anos de 2014 a 2019. As buscas foram realizadas no mês de novembro de 2019.

Foram estabelecidos os critérios de elegibilidade: artigos primários, que abordassem especificamente a temática sobre termorregulação de RN prematuro e os cuidados que podem ser aplicados pela enfermagem. Os critérios de exclusão foram: títulos e resumos que não correspondem à pesquisa, artigos repetidos, artigos de reflexão, resenhas, revisões de literatura, editoriais, teses, dissertações, protocolos de pesquisas e estudos que não abordassem a temática relevante ao alcance do objetivo da revisão.

Após a geração dos resultados da busca, realizou-se a seleção dos estudos primários, de acordo com a questão norteadora e os critérios de inclusão. Todos os estudos foram inicialmente avaliados por meio da análise dos títulos, posteriormente leitura dos resumos e por fim a leitura do texto na íntegra. Foi feito um quadro com catalogação dos artigos, com informações dos autores, tipo de estudo, país do local de estudo, ano de publicação, participantes do estudo, objetivo e principais resultados.

A análise dos dados extraídos dos artigos foi realizada de forma descritiva, possibilitando observar, contar, descrever e classificar os dados, com o intuito de reunir o conhecimento produzido sobre o tema explorado na revisão. As demais etapas serão apresentadas com a categorização dos artigos com base nas evidências, discussão das categorias formadas a partir das leituras dos textos e a apresentação da revisão e síntese do conhecimento.

\section{RESULTADOS}

Utilizando as estratégias de seleção dos estudos, a busca nas bases de dados resultou em 120 itens, cujos títulos repetidos foram excluídos, restando 61 registros para a leitura dos títulos e resumos. Após consenso dos juízes, foram selecionados 12 para análise na 
íntegra. Após a leitura do texto completo, três artigos foram excluídos por apresentarem informações insuficientes acerca do objeto de estudo da presente revisão, assim, incluiu-se nove itens que atenderam aos critérios de elegibilidade.

Os artigos selecionados e analisados retrataram aspectos da atenção de enfermagem na termorregulação de RN prematuros em diferentes países, dois nos Estados Unidos, e um no Brasil, Indonésia, Turquia, Zâmbia, Finlândia, China e um estudo multicêntrico com dados de hospitais de diferentes países. Os anos de publicação variaram de 2014 a 2018, ocorrendo quatro artigos em 2017, três em 2014, um artigo no ano de 2018 e outro em 2015. Com relação ao tipo de abordagem, oito artigos são quantitativos e um artigo é qualitativo. Somando as amostras de todos os artigos, no geral há um $n=2.275$ (93\%) de RN prematuros e um $n=171(7 \%)$ de participantes adultos. O Quadro 1 apresenta os estudos que fizeram parte da análise para a construção da revisão integrativa.

Quadro 1 - Catalogação dos estudos incluídos na síntese para a análise. Macapá, AP, Brasil, 2019 (continua)

\begin{tabular}{|c|c|c|c|c|c|}
\hline Autores e ano & País & $\begin{array}{l}\text { Desenho de } \\
\text { estudo }\end{array}$ & $\begin{array}{l}\text { Tamanho da } \\
\text { amostra }\end{array}$ & Objetivo & $\begin{array}{l}\text { Resultados } \\
\text { principais }\end{array}$ \\
\hline $\begin{array}{l}\text { CALDAS e } \\
\text { colaboradores, } \\
2018^{(15)}\end{array}$ & Brasil & $\begin{array}{l}\text { Estudo de } \\
\text { intervenção e } \\
\text { retrospectivo }\end{array}$ & $\begin{array}{l}475 \\
\text { prematuros }\end{array}$ & $\begin{array}{l}\text { Avaliar a eficácia } \\
\text { de um pacote de } \\
\text { termorregulação } \\
\text { na prevenção } \\
\text { da hipotermia } \\
\text { na admissão em } \\
\text { prematuros. }\end{array}$ & $\begin{array}{l}\text { Redução na } \\
\text { incidência de } \\
\text { hipotermia após } \\
\text { a continuação da } \\
\text { implementação do } \\
\text { protocolo. }\end{array}$ \\
\hline $\begin{array}{l}\text { HANDHAYANTI; } \\
\text { RUSTINA; } \\
\text { BUDIATI, 2017(16) }\end{array}$ & Indonésia & $\begin{array}{l}\text { Crossover } \\
\text { quantitativo }\end{array}$ & $\begin{array}{l}36 \\
\text { prematuros }\end{array}$ & $\begin{array}{l}\text { Comparar a } \\
\text { temperatura } \\
\text { em prematuros } \\
\text { durante } \\
\text { procedimentos } \\
\text { invasivos em } \\
\text { incubadoras ou } \\
\text { em aquecedores } \\
\text { radiantes }\end{array}$ & $\begin{array}{l}\text { O aquecedor } \\
\text { radiante evitou } \\
\text { hipotermia durante } \\
\text { procedimentos } \\
\text { invasivos. No } \\
\text { entanto, é } \\
\text { desaconselhável o } \\
\text { uso rotineiro pois } \\
\text { pode aumentar a } \\
\text { perda insensível de } \\
\text { água. }\end{array}$ \\
\hline $\begin{array}{l}\text { HARER e } \\
\text { colaboradores, } \\
2017^{(17)}\end{array}$ & $\begin{array}{l}\text { Estados } \\
\text { Unidos }\end{array}$ & $\begin{array}{l}\text { Implementação } \\
\text { de intervenção }\end{array}$ & $\begin{array}{l}325 \\
\text { prematuros }\end{array}$ & $\begin{array}{l}\text { Reduzir a taxa } \\
\text { de hipotermia na } \\
\text { admissão pré- } \\
\text { termo }\end{array}$ & $\begin{array}{l}\text { O uso de } \\
\text { uma diretriz } \\
\text { multidisciplinar } \\
\text { resultou em } \\
\text { uma diminuição } \\
\text { de bebês } \\
\text { hipotérmicos. }\end{array}$ \\
\hline $\begin{array}{l}\text { HU e } \\
\text { colaboradores, } \\
2017^{(19)}\end{array}$ & China & $\begin{array}{l}\text { Ensaio Clínico } \\
\text { Randomizado }\end{array}$ & $\begin{array}{l}108 \\
\text { prematuros }\end{array}$ & $\begin{array}{l}\text { Determinar se } \\
\text { a colocação do } \\
\text { RN em sacos } \\
\text { plásticos durante o } \\
\text { transporte reduz a } \\
\text { hipotermia. }\end{array}$ & $\begin{array}{l}\text { A colocação de } \\
\text { bebês em sacos de } \\
\text { polietileno durante } \\
\text { o transporte reduz } \\
\text { a ocorrência de } \\
\text { hipotermia. }\end{array}$ \\
\hline
\end{tabular}




\begin{tabular}{|c|c|c|c|c|c|}
\hline $\begin{array}{l}\text { LEWIS; } \\
\text { JACOBSON, } \\
2017^{(20)}\end{array}$ & $\begin{array}{l}\text { Estados } \\
\text { Unidos }\end{array}$ & $\begin{array}{l}\text { Levantamento } \\
\text { e metodologia } \\
\text { correlacional } \\
\text { descritiva }\end{array}$ & $\begin{array}{l}164 \\
\text { prematuros }\end{array}$ & $\begin{array}{l}\text { Identificar os } \\
\text { procedimentos } \\
\text { de enfermagem } \\
\text { que perturbam } \\
\text { o ambiente } \\
\text { térmico ao abrir a } \\
\text { incubadora de RN } \\
\text { prematuros }\end{array}$ & $\begin{array}{l}\text { A frequência de } \\
\text { procedimentos } \\
\text { de enfermagem } \\
\text { documentados } \\
\text { que perturbam } \\
\text { o ambiente } \\
\text { térmico ao abrir a } \\
\text { incubadora pode } \\
\text { ter efeito negativo } \\
\text { na mudança de } \\
\text { peso. }\end{array}$ \\
\hline $\begin{array}{l}\text { REILLY e } \\
\text { colaboradores, } \\
2015^{(21)}\end{array}$ & Vários & $\begin{array}{l}\text { Ensaio Clínico } \\
\text { Randomizado } \\
\text { (multicêntrico) }\end{array}$ & $\begin{array}{l}813 \\
\text { prematuros }\end{array}$ & $\begin{array}{l}\text { Determinar } \\
\text { se a aplicação } \\
\text { do envoltório } \\
\text { oclusivo aplicado } \\
\text { imediatamente } \\
\text { após o nascimento } \\
\text { reduz a } \\
\text { mortalidade em } \\
\text { bebês prematuros }\end{array}$ & $\begin{array}{l}\text { A aplicação do } \\
\text { envoltório oclusivo } \\
\text { após o nascimento } \\
\text { resulta em maior } \\
\text { temperatura } \\
\text { corporal média, } \\
\text { mas não reduz a } \\
\text { mortalidade. }\end{array}$ \\
\hline $\begin{array}{l}\text { ÇAGLAR; } \\
\text { GÖZEN; INCE, } \\
\text { 2014(22) }\end{array}$ & Turquia & $\begin{array}{l}\text { Ensaio Clínico } \\
\text { Randomizado }\end{array}$ & $\begin{array}{l}59 \\
\text { prematuros }\end{array}$ & $\begin{array}{l}\text { Analisar os efeitos } \\
\text { do uso de sacos } \\
\text { de isolamento de } \\
\text { vinil ou envoltório } \\
\text { de polietileno } \\
\text { para a prevenção } \\
\text { de hipotermia em } \\
\text { prematuros }\end{array}$ & $\begin{array}{l}\text { O } \\
\text { acondicionamento } \\
\text { de prematuros } \\
\text { em sacos de vinil } \\
\text { imediatamente } \\
\text { após o nascimento } \\
\text { está associado } \\
\text { a menores } \\
\text { incidências de } \\
\text { hipotermia. }\end{array}$ \\
\hline $\begin{array}{l}\text { LUNZE e } \\
\text { colaboradores, } \\
2014^{(23)}\end{array}$ & Zâmbia & $\begin{array}{l}\text { Estudo } \\
\text { qualitativo com } \\
\text { grupos focais }\end{array}$ & $\begin{array}{l}171 \\
\text { participantes }\end{array}$ & $\begin{array}{l}\text { Caracterizar } \\
\text { práticas, atitudes } \\
\text { e crenças de } \\
\text { prevenção e } \\
\text { tratamento da } \\
\text { hipotermia }\end{array}$ & $\begin{array}{l}\text { Compreender e } \\
\text { abordar as práticas } \\
\text { comunitárias } \\
\text { de prevenção e } \\
\text { tratamento da } \\
\text { hipotermia podem } \\
\text { ajudar a melhorar } \\
\text { a sobrevivência do } \\
\text { RN. }\end{array}$ \\
\hline $\begin{array}{l}\text { RAISKILA e } \\
\text { colaboradores, } \\
2014^{(24)}\end{array}$ & Finlândia & $\begin{array}{l}\text { Coorte } \\
\text { retrospectiva }\end{array}$ & $\begin{array}{l}295 \\
\text { prematuros }\end{array}$ & $\begin{array}{l}\text { Avaliar as } \\
\text { tendências nas } \\
\text { práticas de cuidado } \\
\text { centrado na família }\end{array}$ & $\begin{array}{l}\text { Os cuidados } \\
\text { hospitalares de } \\
\text { bebês prematuros } \\
\text { apoiam o } \\
\text { envolvimento } \\
\text { dos pais. } \mathrm{Na} \\
\text { avaliação, o ganho } \\
\text { de peso de bebês } \\
\text { melhorou. }\end{array}$ \\
\hline
\end{tabular}

De acordo com os dados obtidos na síntese dos artigos, podemos observar a formação de um conjunto de cuidados no processo de termorregulação do RN, que podem ser realizados por enfermeiros, descrito no Quadro 2. 
Quadro 2 - Cuidados de enfermagem na termorregulação, identificados pela síntese dos artigos. Macapá, AP, Brasil, 2019

\begin{tabular}{|c|c|c|c|c|}
\hline \multirow[t]{2}{*}{ Cuidados de enfermagem } & \multirow[t]{2}{*}{ Objetivo } & \multicolumn{3}{|c|}{ Momento de realização } \\
\hline & & $\begin{array}{l}\text { Pós- } \\
\text { parto }\end{array}$ & $\begin{array}{l}\text { Procedi- } \\
\text { mentos }\end{array}$ & Internação \\
\hline $\begin{array}{l}\text { Não abrir a incubadora } \\
\text { demasiadamente. }\end{array}$ & $\begin{array}{l}\text { Evitar da perda de calor corporal para o } \\
\text { ambiente, prevenindo a hipotermia }\end{array}$ & & $x$ & $x$ \\
\hline $\begin{array}{l}\text { Ajuste da temperatura do } \\
\text { ambiente }\end{array}$ & $\begin{array}{l}\text { Promover uma temperatura ambiente em } \\
\text { uma faixa que não contribua para perda } \\
\text { de calor corporal. }\end{array}$ & $x$ & $x$ & $x$ \\
\hline $\begin{array}{l}\text { Monitoramento da } \\
\text { temperatura do prematuro }\end{array}$ & $\begin{array}{l}\text { Identificar indicativos de hipotermia e } \\
\text { hipertermia, pois tais condições podem } \\
\text { comprometer processos metabólicos e } \\
\text { fisiológicos. }\end{array}$ & & $x$ & $x$ \\
\hline $\begin{array}{l}\text { Utilização da cama } \\
\text { aquecida ou aquecedor } \\
\text { radiante }\end{array}$ & $\begin{array}{l}\text { Proteger da hipotermia ao realizar } \\
\text { procedimentos fora da incubadora, } \\
\text { devendo ser usado com cautela para não } \\
\text { ocorrer hipertermia, ressecamento da } \\
\text { pele e perda de liquido. }\end{array}$ & & $x$ & \\
\hline $\begin{array}{l}\text { Utilização de métodos de } \\
\text { isolamento corporal, como } \\
\text { sacos de vinil, polietileno } \\
\text { ou poliuretano }\end{array}$ & $\begin{array}{l}\text { Diminuir a perda de calor do prematuro } \\
\text { para o ambiente }\end{array}$ & $x$ & & \\
\hline $\begin{array}{l}\text { Estímulo de medidas de } \\
\text { baixo custo para locais } \\
\text { subdesenvolvidos com } \\
\text { recurso limitados }\end{array}$ & $\begin{array}{l}\text { Oferecer alternativas para manter a } \\
\text { cadeia quente, como incentivo ao } \\
\text { contato pele a pele, manter prematuro } \\
\text { coberto, aquecer o ambiente, etc. }\end{array}$ & $x$ & $x$ & $x$ \\
\hline $\begin{array}{l}\text { Avaliar e discutir com a } \\
\text { equipe multiprofissional } \\
\text { os cuidados e tecnologias } \\
\text { adequadas para a } \\
\text { termorregulação do RN } \\
\text { prematuro }\end{array}$ & $\begin{array}{l}\text { Prever qual o melhor cuidado e } \\
\text { dispositivos para termorregulação de } \\
\text { acordo com os protocolos da instituição e } \\
\text { as melhores evidências científicas }\end{array}$ & $x$ & $x$ & $x$ \\
\hline $\begin{array}{l}\text { Registro de saúde correto } \\
\text { nos prontuários }\end{array}$ & $\begin{array}{l}\text { Identificar quais os procedimentos estão } \\
\text { perturbando a termorregulação do RN e } \\
\text { servir de indicadores de qualidade para } \\
\text { melhoria de processos }\end{array}$ & $x$ & $x$ & $x$ \\
\hline
\end{tabular}

\section{DISCUSSÃO}

Após a leitura dos estudos, e levando em consideração os objetivos da revisão de analisar o contexto dos cuidados de enfermagem e as tecnologias para a termorregulação neonatal, ressaltando os principais resultados encontrados a fim de sintetizá-los e direcionando os resultados a uma prática fundamentada em conhecimento científico, emergiram temas comuns organizados em duas categorias: "Cuidados na termorregulação de recém-nascidos prematuros" e "Protocolos para a termorregulação neonatal na prática clínica". 


\section{Cuidados na termorregulação de recém-nascidos prematuros}

Tendo em vista os cuidados de enfermagem frente ao controle de temperatura do RN pré-termo, é importante ressaltar que seus cuidados podem ser tanto benéficos quanto danosos ao quadro de termorregulação. $O$ exemplo dessa relação entre as ações da enfermagem com a alteração do ambiente térmico é percebido em um estudo(19) onde houve um número de 51 procedimentos de cuidados de enfermagem que perturbaram o ambiente térmico ao abrir a incubadora de RN prematuros, tendo a avaliação de sinais vitais como responsável por mais de $90 \%$ das ações que perturbam o ambiente térmico ao abrir a incubadora.

Além do mais, foi comparada a perda de calor dos RN antes e após procedimentos invasivos que envolviam punção venosa realizada com a incubadora aberta. Um artigo ${ }^{(16)}$ constatou queda na temperatura corporal dos prematuros após o tratamento invasivo na incubadora aberta. Destarte, esse quadro pode resultar em baixa estabilidade térmica de prematuros. Além disso, evidenciou-se que os procedimentos de enfermagem que perturbam o ambiente térmico ao abrir a incubadora podem ter um efeito negativo na mudança de peso dos prematuros ${ }^{(19)}$.

Ao analisar os estudos, encontrou-se alternativas à perda de calor ocasionada pela abertura da incubadora, como a utilização da cama aquecida ou aquecedor radiante, quando o prematuro é movido de uma incubadora para um leito mais quente para a realização dos cuidados. Nesse sentido, o crescimento e a termorregulação dos RN mostraram-se ainda melhores do que o tempo permanecido na incubadora ${ }^{(23)}$. Além disso, foi observada elevação da temperatura corporal média após os procedimentos realizados sob aquecedor radiante ${ }^{(16)}$. Assim, sugere que o uso de um aquecedor radiante é um tratamento de enfermagem adequado para proteger as crianças da hipotermia.

Observando o panorama do envolvimento dos pais no cuidado neonatal, também foi perceptível vantagens ao utilizar a cama aquecida, que, além de permitir práticas de cuidados menos invasivas, acarreta maior proximidade entre pais e filhos. A incubadora pode ser um obstáculo para o cuidado pele a pele, já com a cama aquecida pode ser mais fácil iniciar o contato pele a pele sem a necessidade de remover o bebê do leito. Além disso, os pais percebem menos separação do bebê que está em uma cama aquecida, pois tocar e falar com o bebê se torna mais disponível sem a barreira física da parede da incubadora ${ }^{(23)}$.

Em um estudo, foi realizado um conjunto de intervenções para prevenção da hipotermia em prematuros, entre eles encontra-se o uso de berço com fonte de calor radiante, permanentemente ajustada entre 35 e $36^{\circ} \mathrm{C}$, mas nessa ocasião não foi utilizado o colchão térmico; mesmo assim, foi alcançada uma redução na taxa de hipotermia, demonstrando que a ausência desse recurso não interferia diretamente no sucesso da intervenção ${ }^{(15)}$. Em outro, foi estabelecido nas diretrizes que o colchão exotérmico devia ser utilizado em todos os RN abaixo de 35 semanas, e isso resultou em uma redução significativa nas taxas de hipotermia(16).

Do mesmo modo, a enfermagem deve estar alerta para que as intervenções realizadas para prevenir a hipotermia não causem o problema oposto, a hipertermia, exigindo assim que o enfermeiro realize um correto monitoramento do RN. Nessa perspectiva, durante a síntese pôde-se observar que em um estudo ${ }^{(15)}$ ocorreram 11 casos de hipertermia após as medidas de intervenção, já em outro, houve um aumento das taxas de hipertermia de $2 \%$ para $7 \%$ e, com isso, tomaram medidas para diminuir a temperatura da incubadora de transporte e remover o colchão exotérmico se a temperatura dos RN fosse $>37^{\circ} \mathrm{C}^{(17)}$. Sendo assim, ao combinar as diversas formas de termorregulação, é necessário um monitoramento rigoroso das temperaturas dos prematuros para evitar hipertermia.

Apesar dos dispositivos de aquecimento serem ótimas alternativas para que os enfermeiros realizem procedimentos com o RN fora da incubadora, o seu uso de forma inadequada e sem o monitoramento apropriado pode levar a resultados indesejados, como a hipertermia. Como consequência somada a isso, podem causar a evaporação de fluidos, 
que faz com que os bebês sofram ressecamento da pele e pode levar à perda insensível de água(16).

Além disso, o enfermeiro deve estar atento ao ambiente desde o acontecimento do parto, ciente do impacto da diferença entre a temperatura do ambiente intra e extrauterino para o neonato. A exemplo disso, identificou-se que uma baixa temperatura da sala de parto afetou a temperatura média do RN na admissão na unidade neonatal, autores evidenciaram correlação significativa entre a temperatura da sala de parto e a temperatura do prematuro na admissão ${ }^{(17)}$. Apesar desses autores não recomendarem uma temperatura ambiente ideal, no contexto do Brasil, a Portaria 371 de 2014, que instituiu Diretrizes para a organização da atenção integral e humanizada ao RN no Sistema Único de Saúde, recomenda que a temperatura ambiente esteja a $26^{\circ} \mathrm{C}$ para evitar a perda de calor ${ }^{(18)}$.

Uma das possibilidades de cuidados de enfermagem no controle da temperatura corporal é a utilização de métodos de barreiras que visam aquecer o RN através do isolamento, que diminui a perda de calor do prematuro para o ambiente. Nesse sentindo, foram analisados os efeitos do uso de sacos de isolamento de vinil ou envoltório de polietileno para a prevenção de hipotermia em prematuros e verificado que os métodos de barreira na sala de parto e transporte são necessários para manter a temperatura corporal de prematuros ${ }^{(19,21,22)}$. No entanto, apesar desse recurso, evidenciou baixa utilização de algum método de barreira ${ }^{(17)}$.

É interessante destacar os aspectos da assistência ao nascimento no contexto de locais subdesenvolvidos, onde existe carência de tecnologias e profissionais capacitados para realização de uma melhor termorregulação dos prematuros. Sobre essa realidade, em uma investigação etnográfica qualitativa ${ }^{(23)}$ realizada na zona rural da Zâmbia, foi revelado que os profissionais de saúde estão cientes do perigo da hipotermia neonatal, no entanto, a cadeia quente recomendada pela Organização Mundial de Saúde como padrão de atendimento não foi mantida consistentemente durante as primeiras horas após o parto, quando os RN correm maior risco. Sendo assim, recomenda-se compreender e abordar as práticas de prevenção e tratamento da hipotermia para ajudar a melhorar a sobrevivência do $\mathrm{RN}$ em locais com recursos limitados.

\section{Protocolos para a termorregulação neonatal na prática clínica}

Na presente categoria temática, é abordada a importância do cuidado padronizado, principalmente por meios de protocolos e diretrizes essencialmente baseados nas melhores evidências cientificas disponíveis, visando uma melhor interação entre a enfermagem e a equipe multidisciplinar e melhores desfechos neonatais. A padronização do cuidado baseado em evidências requer uma educação extensa e contínua das equipes, com apresentação periódica dos resultados, melhorando a adesão às práticas e aumentando as taxas de melhora da hipotermia(15).

Tendo isso em vista, destacam-se estudos para implementação de diretrizes multidisciplinares e programa de medidas voltadas para a prevenção da hipotermia e melhoria das temperaturas de prematuros internados. Para atingir o propósito, utilizaram a metodologia de planejar-fazer-estudar-agir (PDSA)(17), e também avaliaram a eficácia através de dados antes e após intervenção(15). Ambos estudos focados em padronização dos cuidados obtiveram redução na incidência de hipotermia após as intervenções ${ }^{(15,17)}$.

As intervenções de termorregulação dos prematuros padronizadas avaliadas nos estudos foram: colocação em um berço com fonte de calor radiante ajustada entre 35 e $36^{\circ} \mathrm{C}$; obtenção de uma temperatura axilar de 10 minutos; manter as portas da sala sempre fechadas; aumento da temperatura da sala de operações; uso de sacola de polietileno para o corpo do RN; cobrir a cabeça com uma touca de plástico e uma touca de algodão após secar a região da fontanela; manutenção da temperatura na sala de ressuscitação entre 24 e $27^{\circ} \mathrm{C}$; e transporte para a unidade de admissão hospitalar em uma incubadora aquecida e ajustada a uma temperatura de 35 a $37^{\circ} \mathrm{C}^{(15,17)}$. 
É interessante que os protocolos e diretrizes criados sejam distribuídos a todos os profissionais da unidade neonatal, incluindo enfermeiros e médicos obstetras, e que sejam realizadas sessões educacionais para a troca do conhecimento. Autores afirmam também que, durante a fase de planejamento do estudo, o aspecto multidisciplinar nos permite apresentar estudos anteriores sobre a temperatura da sala de parto e os resultados neonatais à equipe obstétrica, resultando em discussões produtivas para a segurança do paciente e ótimos resultados ao instituir um método padrão de termorregulação ${ }^{(17)}$.

Em síntese, os dois principais estudos da presente categoria temática mostraram que, antes de projetos de intervenção, foram observados valores altos de hipotermia na admissão neonatal, ocorrendo uma diminuição após a implementação dos projetos e diretrizes multidisciplinares, além de não resultar em aumento significativo nas taxas de hipertermia. Ademais, enfatizaram que são necessárias muitas outras práticas para eliminar a hipotermia em bebês prematuros, visando reduzir a taxa para menos de $10 \%^{(15,17)}$.

Já no contexto de países com recursos limitados, são necessárias estratégias diferentes para a implementação de modelos padrões para os cuidados quanto à termorregulação neonatal, como compreender e abordar as práticas comunitárias de prevenção e tratamento da hipotermia, implementação de cuidados pele a pele, treinar os membros da família para apoiar mães no fornecimento de termoproteção e o uso de aquecedores adequados e de baixo custo para prevenir e controlar a hipotermia de bebês e, assim, ajudar a melhorar a sobrevivência do $\mathrm{RN}^{(23)}$.

Salienta-se também o correto registro de saúde nos prontuários como ferramenta importante no cuidado de enfermagem, tanto para a análise da evolução do RN quanto para uma melhor assistência multiprofissional. A falta de documentação completa dos procedimentos que perturbam a termorregulação dos prematuros, como de frequência discreta de abertura da incubadora, limita a capacidade de usar os dados de registro de saúde para determinar resultados sensíveis à enfermagem e outros indicadores de qualidade e segurança para melhoria de processos ${ }^{(15,20)}$.

As limitações desta revisão são referentes à variabilidade dos tipos de pesquisa e de contextos de realização da coleta de dados, o que possivelmente leva à reflexão sobre as nuances da assistência de enfermagem neonatal no cenário brasileiro.

\section{CONSIDERAÇÕES FINAIS}

A partir dos resultados obtidos pela análise dos artigos, evidencia-se que a enfermagem pode realizar procedimentos que afetam negativamente a termorregulação dos prematuros ao abrir a incubadora, contudo, existem cuidados auxiliares, como a utilização de cama aquecida, aquecedor radiante, métodos de isolamento corporal e ajuste da temperatura ambiente. No entanto, o enfermeiro deve ficar atento com esses cuidados, pois um maior aquecimento pode acarretar em hipertermia, ressecamento da pele e perda insensível de água. Em lugares com recursos limitados, onde não há esses dispositivos, recomenda-se compreender e abordar as práticas de prevenção e tratamento da hipotermia para ajudar a melhorar a sobrevivência do RN.

Ademais, foi abordada a questão do cuidado padronizado através da implementação de diretrizes e protocolos e, para isso, exige-se uma educação extensa e contínua das equipes visando uma melhor termorregulaçã̃o. Os protocolos e diretrizes implementados por projetos de intervenção devem ser distribuídos a toda equipe multiprofissional que presta assistência aos prematuros. Na presente revisão, evidenciou-se que, antes de projetos de intervenção, havia altas taxas de hipotermia nos RN, ocorrendo uma diminuição após a implementação.

Portanto, considerando a assistência de enfermagem, os resultados obtidos através 
da literatura científica pertinente permitiram ampliar o conhecimento acerca do cuidado na termorregulação do neonato, as precauções na utilização de tecnologias de termoproteção e, por fim, o papel do enfermeiro inserido em uma equipe multiprofissional que atue de forma normatizada. Contudo, verificou-se a necessidade de mais estudos nacionais a fim de compreender o quadro do cuidado de enfermagem na termorregulação de prematuros no Brasil.

\section{REFERÊNCIAS}

1. Almeida MFB de, Guinsburg R, Sancho GA, Rosa IRM, Lamy ZC, Martinez FE, et al. Hypothermia and early neonatal mortality in preterm infants. J Pediatr. [Internet]. 2014 [acesso em 17 out 2019]; 164(2). Disponível em: https://dx.doi.org/10.1016/j.jpeds.2013.09.049.

2. Ministério da Saúde (BR). Secretaria de Atenção à Saúde. Departamento de Ações Programáticas e Estratégicas. Atenção à saúde do recém-nascido: guia para os profissionais de saúde. Brasília: Ministério da Saúde; 2011.

3. Ricci SS. Enfermagem: materno, neonatal e saúde da Mulher. 3. ed. Rio de Janeiro: Guanabara Koogan; 2015.

4. Ministério da Saúde (BR). Secretaria de Atenção à Saúde. Departamento de Ações Programáticas Estratégicas. Atenção humanizada ao recém-nascido: método canguru: manual técnico. 3. ed. Brasília: Editora do Ministério da Saúde; 2017.

5. Ringer SA. Core concepts: thermoregulation in the newborn, Part II: prevention of aberrant body temperature. Neoreviews. [Internet]. 2013 [acesso em 18 out 2019]; 14(5). Disponível em: https://dx.doi. org/10.1542/neo.14-5-e221.

6. Sousa DS, Sousa Júnior AS, Santos ADR, Melo EV, Lima SO, Almeida-Santos MA, et al. Morbidity in extreme low birth weight newborns hospitalized in a high risk public maternity. Rev. Bras. Saúde Mater. Infant. [Internet]. 2017 [acesso em 18 out 2019]; 17(1). Disponível em: http://dx.doi.org/10.1590/180693042017000100008.

7. Rolim KMC, Freitas LC, Lima A de HG, Magalhães FJ, Gurgel E de PP. Cobertura de polietileno para manutenção da temperatura corporal do recém-nascido. Rev. Enf. Ref. [Internet]. 2015 [acesso em 17 out 2019]; serIV(6). Disponível em: http://dx.doi.org/10.12707/RIV14070.

8. Ministério da Saúde (BR). Atenção humanizada ao recém-nascido: método canguru: manual técnico. Brasília: Ministério da Saúde; 2012.

9. Costa R, Locks MOH, Klock P. Acolhimento na unidade neonatal: percepção da equipe de enfermagem. Rev enferm UERJ [Internet]. 2012 [acesso em 19 out 2019]; 20(3). Disponível em: https:// www.e-publicacoes.uerj.br/index.php/enfermagemuerj/article/view/2382.

10. Conselho Federal de Enfermagem. Portaria $n^{\circ} 2.068 / 2016$. Institui diretrizes para a organização da atenção integral e humanizada à mulher e ao recém-nascido no Alojamento Conjunto. Diário Oficial da União [Internet]. 2016 [acesso em 18 out 2019]. Disponível em: http://bvsms.saude.gov.br/bvs/saudelegis/ $\mathrm{gm} / 2016 /$ prt2068 $21 \quad 10$ 2016.html.

11. Ganong LH. Integrative reviews of nursing research. Res Nurs Health [Internet].1987 [acesso em 18 mar 2019]; 10(1). Disponível em: https://doi.org/10.1002/nur.4770100103.

12. Souza MT de, Silva MD da, Carvalho R de. Integrative review: what is it? How to do it? Einstein (Sao Paulo) [Internet]. 2010 [acesso em 10 out 2019]; 8(1). Disponível em: http://dx.doi.org/10.1590/s167945082010 rw1134.

13. Santos CM da C, Pimenta CA de M, Nobre MRC. The PICO strategy for the research question 
construction and evidence search. Rev. Latino-Am. Enfermagem [Internet]. 2007 [acesso em 10 out 2019]; 15(3). Disponível em: https://doi.org/10.1590/S0104-11692007000300023.

14. Moher D, Liberati A, Tetzlaff J, Altman DG, PRISMA Group. Preferred reporting items for systematic reviews and meta-analyses: the PRISMA Statement. Ann Intern Med [Internet]. 2009 [acesso em 10 out 2019]; 151. Disponível em: https://doi.org/10.7326/0003-4819-151-4-200908180-00135.

15. Caldas JP de S, Millen F de C, Camargo JF de, Castro PAC, Camilo AL da F, Marba STM. Effectiveness of a measure program to prevent admission hypothermia in very low-birth weight preterm infants. $\mathrm{J}$ Pediatr (Rio J) [Internet]. 2018 [acesso em 10 nov 2019]; 94(4). Disponível em: https://doi.org/10.1016/j. jped.2017.06.016.

16. Handhayanti L, Rustina $Y$, Budiati T. Differences in Temperature Changes in Premature Infants During Invasive Procedures in Incubators and Radiant Warmers. Compr Child Adolesc Nurs [Internet]. 2017 [acesso em 11 nov 2019]; 40(sup1). Disponível em: http://doi.org/10.1080/24694193.2017.1386977.

17. Harer MW, Vergales B, Cady T, Early A, Chisholm C, Swanson JR. Implementation of a multidisciplinary guideline improves preterm infant admission temperatures. J Perinatol [Internet]. 2017 [acesso em 10 nov 2019]; 37(11). Disponível em: https://dx.doi.org/10.1038/jp.2017.112.

18. Ministério da Saúde (BR). Secretaria de Atenção à Saúde. Portaria n 371, de 7 de maio de 2014. Institui diretrizes para a organização da atenção integral e humanizada ao recém-nascido (RN) no Sistema Único de Saúde (SUS). Diário Oficial da União, 2014.

19. Hu XJ, Wang L, Zheng RY, Lv TC, Zhang YX, Cao Y, et al. Using polyethylene plastic bag to prevent moderate hypothermia during transport in very low birth weight infants: a randomized trial. J Perinatol [Internet]. 2018 [acesso em 10 nov 2019]; 38(4). Disponível em: https://dx.doi.org/10.1038/s41372-0170028-0.

20. Lewis LA, Jacobson AF. Electronic health record documentation of nursing care procedures and change in weight of healthy, moderately premature neonates. Neonatal Netw [Internet]. 2017 [acesso em 11 nov 2019]; 36(6). Disponível em: https://doi.org/10.1891/0730-0832.36.6.348.

21. Reilly MC, Vohra S, Rac VE, Dunn M, Ferrelli K, Kiss A, et al. Randomized trial of occlusive wrap for heat loss prevention in preterm infants. J Pediatr [Internet]. 2015 [acesso em 11 nov 2019]; 166(2). Disponível em: https://dx.doi.org/10.1016/j.jpeds.2014.09.068.

22. Çağlar S, Gözen D, Ince Z. Heat loss prevention (help) after birth in preterm infants using vinyl isolation bag or polyethylene wrap. J obstet gynecol neonatal nurs [Internet]. 2014 [acesso em 12 nov 2019]; 43(2). Disponível em: https://dx.doi.org/10.1111/1552-6909.12291.

23. Lunze K, Yeboah-Antwi K, Marsh DR, Kafwanda SN, Musso A, Semrau K, et al. Prevention and management of neonatal hypothermia in rural Zambia. PLoS One [Internet]. 2014 [acesso em 13 nov 2019]; 9(4). Disponível em: https://dx.doi.org/10.1371\%2Fjournal.pone.0092006.

24. Raiskila S, Axelin A, Rapeli S, Vasko I, Lehtonen L. Trends in care practices reflecting parental involvement in neonatal care. Early hum dev [Internet]. 2014 [acesso em 13 nov 2019]; 90(12). Disponível em: https://dx.doi.org/10.1016/j.earlhumdev.2014.08.010. 
Recebido: 19/12/2019

Finalizado: 21/07/2020

Editora associada: Tatiane Herreira Trigueiro

\author{
Autor Correspondente: \\ Leilson da Silva Lima \\ Universidade Federal do Amapá \\ Rod. Juscelino Kubitschek, km 02, s/n - 68903-419 - Macapá, AP, Brasil \\ E-mail: leilsonsilva9@hotmail.com
}

Contribuição dos autores:

Contribuições substanciais para a concepção ou desenho do estudo; ou a aquisição, análise ou interpretação de dados do estudo - LSL, EAFR, EMS, JPGM

Elaboração e revisão crítica do conteúdo intelectual do estudo - LSL, EAFR, EMS, JPGM

Aprovação da versão final do estudo a ser publicado - LSL

Responsável por todos os aspectos do estudo, assegurando as questões de precisão ou integridade de qualquer parte do estudo - LSL

\title{
cc) (4)
}

Este obra está licenciado com uma Licença Creative Commons Atribuição 4.0 Internacional. 